\title{
The Possible Role of Anti-Epileptic Drugs (AEDS) in Treating Levodopa Induced Dyskinesia (LID)
}

\author{
Mohamed Khateb* \\ *Department of Neurology, Rambam Health Care Campus, Israel \\ *Corresponding author: Mohamed Khateb, Department of Neurology, Rambam Health Care Campus, Haifa, Israel. \\ To Cite This Article: Mohamed Khateb, The Possible Role of Anti-Epileptic Drugs (AEDS) in Treating Levodopa Induced Dyskinesia (LID). Am J \\ Biomed Sci \& Res. 2019 - 6(4). AJBSR.MS.ID.001058. DOI: 10.34297/AJBSR.2019.06.001058.
}

Received: 眥 December 03, 2019; Published: 阱 December 05, 2019

\begin{abstract}
Parkinson's disease (PD) is a common neurodegenerative disease with a lifetime risk of 1-2\%. It primarily affects the basal ganglia system (BG). Disruption of motor functions is one of the main clinical manifestations of PD. The main treatments of PD are L-dopa and dopamine receptor agonists. However, they are usually accompanied with dyskinesia (L-dopa induced dyskinesia or LID) which is common and very difficult to treat causing a significant decrease in patient's quality of life. The exact mechanism beyond LID is largely unclear. A leading theory, based on the classical basal ganglia-cortical loop model, claimed that LID might be the result of dis-inhibition of the motor cortex (mainly the supplementary motor cortex or SMA) because of hypoactivity of GPi. At the cellular level, mechanisms involving pulsatile stimulation of dopamine receptors, dysregulation of genes and proteins in neurons resulting in changes in neural discharge patterns between BG and cortex were reported. Here, we are exploring the possible effects of anti-epileptic drugs (AED) in improving LID. AED are widely used agents mainly for controlling seizure disorders. Moreover, they have been used for other neurological and psychiatrial disorders. Part of the AED were investigated in PD patients with LID. Despite the lack of multiple wide prospective double-blind placebo-controlled trials, the current evidences provide possible positive effects in alleviating LID for at least part of AED such as Levetiracetam and others. We believe future clinical as well as pre-clinical research is recommended for properly investigating the effects of other AED in treating LID.
\end{abstract}

Keywords: Levodopa-induced dyskinesia; Parkinson’s diseases; Anti-epileptic drugs; Motor cortex; Basal ganglia.

Abbreviations: PD: Parkinson's Diseases; BG: Basal Ganglia; LID: Levodopa Induced Dyskinesia; AED: Anti-Epileptic Drugs; GPi: Globus Pallidus internus; SNr: Substantia Nigra pars reticulata; SMA: Supplementary Motor Area; NMDA: N-Methyl-D-Aspartate; AMPA: $\alpha$-amino-3-hydroxy-5methyl-4-isoxazolepropionic acid; GABA: Gamma-Aminobutyric Acid; MAO-B: Monoamine Oxidase B, VA: Ventral Anterior; VL: VentroLateral.

\section{Body}

Parkinson's disease (PD) is a neurodegenerative disorder with a lifetime risk of $1 \%-2 \%$ [1,2]. Patients present with motor as well as non-motor symptoms and signs [1,3,4]. PD main motor signs are tremor, rigidity, bradykinesia, gait instability and more. Non-motor disturbances include sleep, autonomic problems and cognitive decline $[1,4]$. PD is usually treated by drugs that enhance dopaminergic neurotransmission, such as levodopa and dopamine receptor agonists. These anti-parkinsonian drugs markedly improve motor performance, especially at the early stages of the disease. In contrast, in the more advanced stages of PD, patients only partially respond to the presently available pharmacological and neurosurgical treatments and develop severe adverse events to anti-Parkinsonian drugs $[1,5-7,56]$. One important complication of levodopa and dopamine receptor agonists is the levodopa- induced dyskinesia (LID). LID develops in almost all long-standing PD patients treated with levodopa or dopamine receptor agonists, and manifests as involuntary movements induced by the antiParkinsonian drugs $[8,9]$.

LIDs are common and difficult to treat [9]. In general, they can be classified as chorea which is hyperkinetic dance-like movements and dystonia referring to sustained abnormal muscle contractions [10]. Additional motor manifestations in LID include ballism and choreoathetosis [8,51]. With increasing duration of treatment, the frequency as well as the severity of the dyskinesia exacerbate [11]. LID appear in $20-30 \%$ of patients receiving levodopa for less than two years. Over half of patients with PD will develop dyskinesia within six months of treatment [8,10]. Eventually, most (up to $80 \%$ ) of levodopa-treated PD patients has LID within five years $[8,9]$. 
The pathophysiological mechanisms underlying LID are poorly understood. At the network level, LID may be the result of reduced activity of STN and GPi neurons. The decreased activity of GPi causes dis-inhibition to activity in the motor cortex, especially SMA [12,13]. At the cellular level, LID has been associated with pulsatile stimulation of dopamine receptors, dysregulation of genes and proteins in neurons resulting in changes in neural discharge patterns between BG and cortex $[8,14]$. Furthermore, metabolic activity in thalamic nuclei of ventral anterior (VA) and ventrolateral (VL) is significantly decreased in LID [8]. It is lower than that observed in normal and parkinsonian untreated animals. More importantly, it is below than that found in non-dyskinetic, levodopa treated animals [15]. These findings indicate that hypo-activity of BG output nuclei (GPi and SNr) may be related to LID development by inducing hyperactivity of the motor cortex especially the supplementary motor area (SMA). Indeed, electrophysiological evidences proposed that LID is associated with marked decrease in firing frequency of the GPi neurons both in parkinsonian monkeys [8,16] and in PD patients [17]. Furthermore, changes in firing pattern, and not only in firing frequency, may be involved in LID [16]. Both D1 and D2 dopamine receptor agonists disrupt neural firing pattern indicating that irregular firing patterns, more than change in firing rate, are crucial in LID [8].

Therefore, the electrophysiological mechanisms involved in LID include combination of significant decrease in GPi firing rate and a modification of the firing patterns. Mechanisms involving dysfunction of dopamine release, changes in dopamine receptor signaling and changes in non-dopamine systems were described as well [18-20]. Many strategies tried to treat LID such as opoidreceptors antagonist [21], NMDA and AMPA receptors antagonists [22-25], H3 Histamine receptor antagonists, adenosine A2a receptors antagonists [26] and more with little tangible success. Interestingly, Anti-epileptic drugs (AEDs) were also tried in order to treat LID and despite the relatively small amount of studies, promising effects may exist.

Anti-epileptic drugs (AEDs) are widespread medications affecting neural activity at the cellular as well as network level by diverse mechanisms. The exact mechanism of action of part of them is largely unknown. Besides treating epilepsy, they have been widely used for other purposes like in major psychiatric disorders, migraine and more [27-31]. The motivation beyond using AEDs for treating LIDs rises from electrophysiological findings discussed above including changes in firing rate like hypoactivation of GPi and dis-inhibition of motor cortex in LID. Theoretically, AEDs may aid reversing the dis-inhibited motor cortex in LID. Interestingly, most of the evidences about the effectiveness of AEDs in LID has been investigated mainly for second-generation drugs, maybe because their better safety profile. There are very little studies about firstgeneration drugs like phenytoin, carbamazepine, etc.
Levetiracetam is a relatively well-tolerated AED with a poorly understood mechanism of action. Most likely is has more than one potential mechanism of action such as synaptic vesicle $2 \mathrm{~A}$ modulation, modulating voltage-gated calcium channels [32] and potassium channels [33]. In LID, it has been shown to abolish the abnormal movements in rat models of LID and MPTP-lesioned macaques in a dose-dependent pattern [34-36]. Few clinical trials investigated the efficacy of levetiracetam in treating LID. A randomized placebo- controlled double-blind pilot study in nine PD patients suggested improvements of these patients reflected by increasing the time free from dyskinesia [36,37]. In addition, wider multicenter double-blind placebo- controlled trial in 38 LID patients demonstrated a significant decrease in dyskinesia time with levetiracetam at doses of 500 and $1000 \mathrm{mg}$ per day [38]. Pre-clinical animal studies and additional case reports indicate that Levetiracetam may reduce other types of dyskinesia rather than LID such as fever- induced chorea as well as paroxysmal choreoathetosis [40]. On the other hand, other studies showed that levetiracetam was not well tolerated in PD patients with LID mainly because of its side effects as well as worsening of the dyskinesia [39].

Topiramate acts in many mechanisms including voltage gated sodium channel blockade, modulating GABA and Glutamate synaptic transmission, carbonic anhydrase inhibitor and more [32]. Contradicting evidences were reported about its effect on LID patients. On the one hand, it was demonstrated to reduce LID in rat and non-human primate models $[36,41,42]$. On the other hand, a small-randomized double-blind trial in 15 patients with LID demonstrated that topiramate did not improve the dyskinesia and even exacerbates it [43]. Zonisamide has also multiple mechanisms of action including voltage gated sodium and calcium channels blockade, inhibiting of glutamate release, increase in dopamine synthesis, MAO-B inhibition and more [32]. A notable doubleblind trial of 422 patients showed that zonisamide improves PD dyskinesia and diminished the time of wearing off [44]. However, the positive effect on LID was not consistent across groups (improvement only in the $50 \mathrm{mg}$ group, but not in the $25 \mathrm{mg}$ and $100 \mathrm{mg}$ groups) [53,54].

Valporate is a first-generation AED. Its mechanism of action is complicated and poorly understood [32]. Few studies in the past examined its efficacy in treating LID concluding that it may yield moderate improvement in the dyskinesia, but this was not significant [48]. Furthermore, previous studies investigated valproate as a treatment for tardive dyskinesia in psychiatric patients with very small effects [49]. Other studies demonstrated no effects [50]. Gabapentin was also investigated and failed to improve LIDs $[53,55]$. Other AEDs were not directly investigated for LID but for other types of dyskinesia. For example, low dose of oxacarbamezapine was shown to reduce Paroxysmal Kinesigenic Dyskinesia in 4 patients [45]. However, Carbamazepine (similar 
to oxacarbamezapine) itself is known to cause dyskinesia, as carbamazepine-induced dyskinesia is the second most common AED induced dyskinesia [46,47]. Lamotrigine also was suggested to be effective in Paroxysmal Kinesigenic Dyskinesias [52].

\section{Conclusion}

To conclude, LID is a common and a difficult to treat complication in PD patients receiving L-dopa or dopamine agonists. Only part of AEDs were directly addressed to improve this dyskinesia. Levetritacem is the drug with the most evidences for treating LID with positive effects in most of the clinical studies. Others were not properly investigated with only small trials lacking statistical power and consistency in findings. Counting on the pathophysiological mechanism, AEDs and especially the voltagegated sodium channels blockers may help alleviate LID by reversing the dis-inhibited motor cortex and the irregular firing patterns of neurons in the motor cortex as well as striatum. Such drugs include phenytoin, Lacosamide, carbamazepine and Lamotrigine. Therefore, we recommend that larger trials will be conducted exploring the real effects of those drugs in LID patients.

\section{Acknowledgements}

Not to mention.

\section{Conflict of Interest}

No conflict of interest.

\section{References}

1. Kalia LV, Kalia SK, Lang AE (2015) Disease-modifying strategies for Parkinson's disease. Mov Disord 30(11): 1442-1450.

2. Ascherio A, Schwarzschild MA (2016) The epidemiology of Parkinson's disease: risk factors and prevention. Lancet Neurol 15(12): 1257-1272.

3. Lanciego JL, Luquin N, Obeso JA (2012) Functional neuroanatomy of the basal ganglia. Cold Spring Harb Perspect Med 2(12): a009621.

4. Calabresi P, Picconi B, Tozzi A, Ghiglieri V, Di Filippo M (2014) Direct and indirect pathways of basal ganglia: a critical reappraisal. Nat Neurosci 17(8): 1022-1030.

5. Nelson AB, Kreitzer AC (2014) Reassessing models of basal ganglia function and dysfunction. Annu Rev Neurosci 37:117-135

6. Goldberg JA, Boraud T, Maraton S, Haber SN, Vaadia E, et al. (2002) Enhanced synchrony among primary motor cortex neurons in the 1-methyl-4-phenyl-1, 2, 3, 6-tetrahydropyridine primate model of Parkinson's disease. J Neurosci 22(11): 4639-4653.

7. Verschuur CV, Suwijn SR, Boel JA, Post B, Bloem BR, et al. (2019) Randomized delayed-start trial of levodopa in Parkinson's disease. New England Journal of Medicine. 380(4): 315-24.

8. Bezard E, Brotchie JM, Gross CE (2001) Pathophysiology of levodopainduced dyskinesia: potential for new therapies. Nat Rev Neurosci 2(8): 577-588.

9. Fabbrini G, Brotchie JM, Grandas F, Nomoto M, Goetz CG (2007) Levodopa-induced dyskinesias. Mov Disord 22(10): 1379-1389.

10. Crossman A (1987) Primate models of dyskinesia: the experimental approach to the study of basal ganglia- related involuntary movement disorders. Neuroscience 21(1): 1-40.

11. Marsden CD (1982) Basal ganglia disease. Lancet 2(8308): 1141-1147.

12. Bezard E, Crossman AR, Gross CE, Brotchie JM (2001) Structures outside the basal ganglia may compensate for dopamine loss in the presymptomatic stages of Parkinson's disease. FASEB J 15(6): 1092-1094.

13. Rascol O, Sabatini U, Chollet F, Celsis P, Montastruc JL, et al. (1992) Supplementary and primary sensory motor area activity in Parkinson's disease: regional cerebral blood flow changes during finger movements and effects of apomorphine. Arch Neurol 49(2): 144- 148.

14. Obeso JA, Olanow CW, Nutt JG (2000) Levodopa motor complications in Parkinson's disease. Trends Neurosci 23(10): S2-S7.

15. Mitchell IJ, Crossman AR, Liminga U, Andren P, Gunne LM (1992) Regional changes in 2-deoxyglucose uptake associated with neuroleptic-induced tardive dyskinesia in the cebus monkey. Mov Disord 7(1): 32-37.

16. Boraud T, Bezard E, Bioulac B, Gross CE (2001) Dopamine agonistinduced dyskinesias are correlated to both firing pattern and frequency alterations of pallidal neurones in the MPTP-treated monkey. Brain 124(3): 546-557.

17. Graybiel AM, Canales JJ, Capper Loup C (2000) Levodopa-induced dyskinesias and dopamine-dependent stereotypies: a new hypothesis. Trends Neurosci 23(10): S71-S77.

18. Feuerstein C, Serre F, Gavend M, Pellat J, Perret J, et al. (1997) Plasma 0-methyldopa in levodopainduced dyskinesias. A bioclinical investigation. Acta Neurol Scand 56(6): 508-524.

19. Montgomery EB (1992) Pharmacokinetics and pharmacodynamics of levodopa. Neurology 42(1): 17-22.

20. De la Fuente Fernandez R, Lu JQ, Sossi V, Jivan S, Schulzer M, et al. (2001) Biochemical variations in the synaptic level of dopamine precede motor fluctuations in Parkinson's disease: PET evidence of increased dopamine turnover. Ann Neurol 49(3): 298-303.

21. Stoessl AJ, Polanski E, Frydryszak H (1993) The opiate antagonist naloxone suppresses a rodent model of tardive dyskinesia. Mov Disord 8(4): 445-452.

22. Papa SM, Chase TN (1996) Levodopa-induced dyskinesias improved by a glutamate antagonist in parkinsonian monkeys. Ann Neurol 39(5): 574578.

23. Blanchet PJ, Konitsiotis S, Chase TN (1998) Amantadine reduces levodopa-induced dyskinesias in parkinsonian monkeys. Mov Disord 13(5): 798-802.

24. Blanchet PJ, Konitsiotis S, Whittemore ER, Zhou ZL, Woodward RM, et al. (1999) Differing effects of N-methyl-Daspartate receptor subtype selective antagonists on dyskinesias in levodopa-treated 1-methyl4-phenyltetrahydropyridine monkeys. J Pharmacol Exp Ther 290(3): 1034-1040.

25. Konitsiotis S, Blanchet PJ, Verhagen L, Lamers E, Chase TN (2000) AMPA receptor blockade improves levodopa-induced dyskinesia in MPTP monkeys. Neurology 54(8): 1589-1595.

26. Kanda T, Jackson MJ, Smith LA, Pearce RK, Nakamura J, et al. (1998) Adenosine A2A antagonist: a novel antiparkinsonian agent that does not provoke dyskinesia in parkinsonian monkeys. Ann Neurol 43(4): 507513.

27. Reuter U, Del Rio MS, Diener HC, Allais G, Davies B, et al. (2009) Migraines with and without aura and their response to preventive therapy with topiramate. Cephalalgia 30(5): 543-551.

28. Hershey, Andrew D, Vockell AL, LeCates S, Kabbouche M (2002) Effectiveness of topiramate in the prevention of childhood headaches. Headache 42(8): 810-818.

29. Von Seggern Randal L, Lisa K Mannix, James U Adelman (2002) Efficacy of topiramate in migraine prophylaxis: a retrospective chart analysis. Headache 42(8): 804-809.

30. Birkhimer L J, J L Curtis, Michael Wong Jann (1985) Use of carbamazepine in psychiatric disorders. Clin Pharm 4(4): 425-434.

31. Elphick Martin (1989) Clinical issues in the use of carbamazepine in psychiatry: a review. Psychol Med 19(3): 591-604. 
32. Devinsky O, Vezzani A, O’Brien T, Jette N, Scheffer IE, et al. (2018) Epilepsy. Nat Rev Dis Primers 4: 18024.

33. Madeja Michael, Margineanu DG, Gorji A, Siep E, Boerrigter P, et al. (2003) Reduction of voltage-operated potassium currents by levetiracetam: a novel antiepileptic mechanism of action?. Neuropharmacology 45(5): 661-671.

34. Bezard Erwan, Hill MP, Crossman AR, Brotchie JM, Michel A, et al. (2004) Levetiracetam improves choreic levodopa-induced dyskinesia in the MPTP- treated macaque. Eur J Pharmacol 485(1-3): 159-164.

35. Hill Michael P, Ravenscroft P, Bezard E, Crossman AR, Brotchie JM, et al. (2004) Levetiracetam potentiates the antidyskinetic action of amantadine in the 1- methyl-4-phenyl-1, 2, 3, 6-tetrahydropyridine (MPTP)-lesioned primate model of Parkinson's disease. J Pharmacol Exp Ther 310(1): 386-394.

36. Vijayakumar Dhanya, Joseph Jankovic (2016) Drug-induced dyskinesia, part 1: treatment of levodopa- induced dyskinesia. Drugs 76(7): 759777.

37. Zesiewicz TA, Sullivan KL, Maldonado JL, Tatum WO, Hauser RA (2005) Open-label pilot study of levetiracetam (Keppra) for the treatment of levodopa-induced dyskinesias in Parkinson's disease. Mov Disord 20(9): 1205-1209.

38. Stathis P, Konitsiotis S, Tagaris G, Peterson D (2011) Levetiracetam for the management of levodopa-induced dyskinesias in Parkinson's disease. Mov Disord 26(2): 264-270.

39. Lyons KE, Pahwa R (2006) Efficacy and tolerability of levetiracetam in Parkinson disease patients with levodopa-induced dyskinesia. Clin Neuropharmacol 29(3): 148-153.

40. Zesiewicz Theresa A, Pathak A, Sullivan KL, Shamayev M, Hauser RA, et al. (2006) Treatment of chorea with levetiracetam. Eur J Clin Pharmacol 62(1): 87.

41. Silverdale MA, Nicholson SL, Crossman AR, Brotchie JM (2005) Topiramate reduces levodopa-induced dyskinesia in the MPTPlesioned marmoset model of Parkinson's disease. Mov Disord 20(4): 403-409.

42. Kobylecki C, Cenci MA, Crossman AR, Ravenscroft P (2010) Calciumpermeable AMPA receptors are involved in the induction and expression of l-DOPA-induced dyskinesia in Parkinson's disease. J Neurochem 114(2): 499-511.

43. Kobylecki C, Burn DJ, Kass Iliyya L, Kellett MW, Crossman AR, et al. (2014) Randomized clinical trial of topiramate for levodopa-induced dyskinesia in Parkinson's disease. Parkinsonism Relat Disord 20(4): 452-455.
44. Murata M, Hasegawa K, Kanazawa I, Fukasaka J, Kochi K, et al. (2015) Zonisamide improves wearing-off in Parkinson's disease: a randomized, double-blind study. Mov Disord 30(10): 1343-1350.

45. Chillag Kipp L, Steven T DeRoos (2009) Oxcarbazepine use in paroxysmal kinesigenic dyskinesia: report on four patients. Pediatr Neurol 40(4): 295-297.

46. Chadwick D, E H Reynolds, C D Marsden (1976) Anticonvulsant-induced dyskinesias: a comparison with dyskinesias induced by neuroleptics. J Neurol Neurosurg Psychiatry 39(12): 1210-1218.

47. Zaatreh Megdad M (2003) Anticonvulsant-induced dyskinesia. Expert Opin Drug Saf 2(4): 385-393.

48. Price P A, J D Parkes, C D Marsden (1978) Sodium valproate in the treatment of levodopa-induced dyskinesia. J Neurol Neurosurg Psychiatry 41(8): 702-706.

49. Chadda R, P Kulhara (1986) A trial of sodium valproate in tardivedyskinesia. Indian J Psychiatry 28(1): 79-82.

50. Nutt John G, Adrian C Williams, Donald B Calne (1980) Effect of sodium valproate on parkinsonism and L-DOPA induced dyskinesia. Brain Research Bulletin 5(2): 589-593.

51. Papavasiliou PS, Gellene R, Cotzias GC (1969) Modification of parkinsonism: Dyskinesias accompanying treatment with dopa. Psychotropic drugs and dysfunctions of the basal ganglia, GE Crane and R. Gardner eds pp.140.

52. Uberall MA, Wenzel D (2000) Effectiveness of lamotrigine in children with paroxysmal kinesigenic choreoathetosis. Dev Med Child Neurol 42(10): 699-700.

53. Bargiotas Panagiotis, Spyridon Konitsiotis (2013) Levodopa-induced dyskinesias in Parkinson's disease: emerging treatments. Neuropsychiatr Dis Treat 9: 1605-1617.

54. Murata M, Hasegawa K, Kanazawa I (2007) Zonisamide improves motor function in Parkinson disease: a randomized, double-blind study Neurology 68(1): 45-50.

55. Van Blercom N, Lasa A, Verger K, Masramon X, Sastre VM, et al. (2004) Effects of gabapentin on the motor response to levodopa: a doubleblind, placebo-controlled, crossover study in patients with complicated Parkinson disease. Clin Neuropharmacol 27(3): 124-128.

56. Assaf Fadi, Yitzhak Schiller (2019) A chemogenetic approach for treating experimental Parkinson's disease. Mov Disord 34(4): 469-479. 